\title{
Searching for the critical point of strongly interacting matter in nucleus-nucleus collisions at CERN SPS
}

\author{
Nikolaos Davis* for the NA61/SHINE collaboration \\ Institute of Nuclear Physics, Polish Academy of Sciences \\ E-mail: nikolaos.daviseifj.edu.pl
}

\begin{abstract}
One of the primary goals of the NA61/SHINE experiment at CERN SPS is the detection of the critical point $(\mathrm{CP})$ of strongly interacting matter. In the interests of this search, an energy (beam momentum $13 \mathrm{~A}-150 \mathrm{~A} \mathrm{GeV/c})$ and system size $(\mathrm{p}+\mathrm{p}, \mathrm{p}+\mathrm{Pb}, \mathrm{Be}+\mathrm{Be}, \mathrm{Ar}+\mathrm{Sc}, \mathrm{Xe}+\mathrm{La})$ scan is being performed.

A number of observables are being considered as possible signatures of the CP. Among these, local fluctuations of the proton density are especially suited for the task, being connected to the critical behavior of the chiral phase transition order parameter in the neighborhood of the CP. In particular, proton density fluctuations are probed by means of an intermittency analysis of the proton second scaled factorial moments (SSFMs) in transverse momentum space, expected by universality theory to scale as a power-law in the vicinity of the CP.

A previous analysis of this sort probed a number of NA49 heavy ion collisions of different size [1]; significant power-law fluctuations were observed in "Si" $+\mathrm{Si}$ collisions at $158 \mathrm{~A} \mathrm{GeV/c}$, with a power-law exponent consistent with the theoretically expected critical value, within uncertainties. Recently, NA61/SHINE Be+Be collisions at $150 \mathrm{~A} \mathrm{GeV/c}$ were similarly probed, yielding a negative result.

We now extend the analysis to NA61/SHINE Ar+Sc collisions at $150 \mathrm{~A} \mathrm{GeV/c}$. The system size and freeze-out baryochemical potential are similar to NA49 "Si"+Si, and preliminary analysis suggests the presence of intermittency. We employ statistical techniques in order to subtract noncritical background present in factorial moments and enhance the signal in cases of low statistics. Through combined use of critical and background Monte Carlo simulations, we assess the quality and statistical significance of the observed intermittency effect.
\end{abstract}

European Physical Society Conference on High Energy Physics - EPS-HEP2019 -

10-17 July, 2019

Ghent, Belgium

${ }^{*}$ Speaker. 


\section{Introduction}

\subsection{The NA61/SHINE experiment}

NA61/SHINE is a fixed target particle and high-energy nuclear physics experiment at CERN SPS [2], colliding a variety of beams on hydrogen and nuclear targets. The main component of its detector is a system of eight Time Projection Chambers (TPCs), some of which are placed inside a strong magnetic field ensuring precise measurements of charged particle momenta. Centrality of collisions is determined through the energy deposit of projectile remnants (spectators) measured in the forward calorimeter.

The physics goals of NA61/SHINE comprise a neutrino, cosmic ray and strong interactions programme. In particular, the strong interactions programme has as one of its stated goals the search for the critical point (CP) of strongly interacting matter. To this purpose, a scan is performed of the phase diagram of strongly interacting matter, varying system size and collision energy, thus probing different freeze-out conditions in temperature $T$ and baryochemical potential $\mu_{B}$ (Fig.1 left). In the present work, we review a number of observables considered as possible experimental signatures of the $\mathrm{CP}$.

\subsection{Critical point observables}
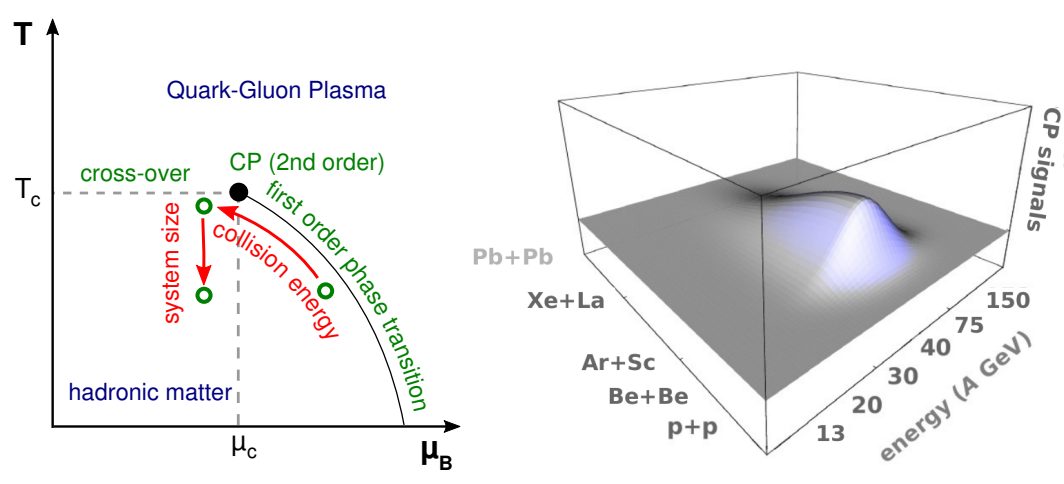

Figure 1: Left: Hypothetical sketch of the phase diagram of strongly interacting matter with critical point, drawn as a function of baryochemical potential $\mu_{B}$ and temperature $T$. Right: Theoretical studies predict the presence of a "hill of fluctuations" as a function of colliding system size and energy, for observables sensitive to the CP (see Ref.[3] for more details).

A characteristic feature of a second order phase transition (expected to occur at the $\mathrm{CP}$ ) is the divergence of the correlation length, leading to a scale-invariant system and an expected "hill" of increased fluctuations in various observables in the $\mathrm{CP}$ vicinity (Fig.1 right). Of particular interest are local power-law fluctuations [4] of the net baryon density, connected to the order parameter of the QCD chiral phase transition, the chiral condensate. At finite baryochemical potential, critical fluctuations are also transferred to the net proton density, as well as to the proton and antiproton densities separately $[5,6,7]$. At the $\mathrm{CP}$, the fluctuations of the order parameter are self-similar [8], belonging to the 3D-Ising universality class, and can be detected in transverse momentum space within the framework of an intermittency analysis $[7,9,10]$ of proton density fluctuations by use of scaled factorial moments (SFMs). A detailed analysis, augmented by properly adapted 
statistical techniques, can be found in Ref. [1], where we study various heavy nuclei collision datasets recorded in the NA49 experiment at maximum energy $\left(158 \mathrm{~A} \mathrm{GeV} / c, \sqrt{s_{N N}} \approx 17 \mathrm{GeV}\right.$ ) of the SPS (CERN).

\section{Method of intermmittency analysis}

Intermittency is defined as the power-law scaling of the Second Scaled Factorial Moments (SSFMs) of protons as a function of bin size in transverse momentum space. The SSFMs are calculated by partitioning a region of transverse momentum space into a lattice of $M \times M$ equalsize bins, and counting the number of proton pairs per bin:

$$
F_{2}(M)=\left\langle\frac{1}{M^{2}} \sum_{i=1}^{M^{2}} n_{i}\left(n_{i}-1\right)\right\rangle /\left\langle\frac{1}{M^{2}} \sum_{i=1}^{M^{2}} n_{i}\right\rangle^{2}
$$

where $n_{i}$ is the number of particles in the $i$-th bin and $M^{2}$ is the total number of bins, and we average over bins and events $(\langle\ldots\rangle)$. In the case of a pure system exhibiting critical fluctuations, $F_{2}(M)$ is expected to scale with $M$, for large values of $M$, as a power-law:

$$
F_{2}(M) \sim M^{2 \phi_{2}}, \phi_{2}=\phi_{2, c r}^{B}={ }^{5} / 6
$$

where $\phi_{2}$ is the intermittency index, and provided the freeze-out occurs at exactly the critical point [7].

Noisy experimental data require the subtraction of a background of uncorrelated \& misidentified protons, which is achieved through the construction of correlation-free mixed events. A correlator $\Delta F_{2}(M)$ can then be defined in terms of the moments of data and mixed events. In the special case where the background dominates over the critical component, Monte Carlo simulations indicate we can approximate the correlator as:

$$
\Delta F_{2}^{(e)}(M) \simeq F_{2}^{(d)}(M)-F_{2}^{(m)}(M),
$$

where mixed event $(m)$ moments are simply subtracted from data $(d)$ moments [1]. $\Delta F_{2}(M)$ should then scale as a power law, $\Delta F_{2}(M) \sim M^{2 \phi_{2}}$, in a limited range, with the same intermittency index as the pure critical system.

Furthermore, calculation of SSFMs is smoothed by averaging over many lattice positions (lattice averaged SSFMs, see Ref. [1]). An improved estimation of statistical errors of SSFMs is achieved by use of the bootstrap method $[11,12,13]$, whereby the original set of events is resampled with replacement [1]. Fitting $\Delta F_{2}^{(e)}(M)$ to obtain $\phi_{2}$ confidence intervals, however, is complicated by bin correlations among $M$-values. Ideally, a correlated fit should be used incorporating information from the bootstrap [11], but such fits are often unstable [14]. The matter is under current investigation.

A proton generating modification of the Critical Monte Carlo (CMC) code [4, 7] is used to simulate a system of critically correlated protons, which are mixed with a non-critical background to study the effects on the quality of intermittency analysis. 


\section{Results}

\subsection{Intermittency analysis: $\Delta F_{2}(M)$}

Proton intermittency analysis was first performed on data collected by the NA49 experiment [1]. Three collisions systems of different size were analyzed: "C" $+\mathrm{C}$, " $\mathrm{Si}$ " $+\mathrm{Si}$ and $\mathrm{Pb}+\mathrm{Pb}$ at midrapidity, at the maximum SPS energy of $158 \mathrm{~A} \mathrm{GeV/c}$. Fig.2(a-c) shows the correlator $\Delta F_{2}(M)$ as a function of bin size $M$ for the analyzed systems. No intermittency was detected in " $\mathrm{C}$ " $+\mathrm{C}$ and $\mathrm{Pb}+\mathrm{Pb}$; by contrast, the "Si" $+\mathrm{Si}$ system exhibits power-law fluctuations compatible with criticality. For the latter, the intermittency index value was estimated, through the bootstrap, as $\phi_{2, B}=0.96_{-0.25}^{+0.38}$ (stat.) \pm 0.16 (syst.) [1].

Motivated by the positive NA49 "Si"+Si result, an intermittency analysis was performed on data collected by the successor NA61/SHINE experiment of two candidate systems, ${ }^{7} \mathrm{Be}+{ }^{9} \mathrm{Be}$ [15] and ${ }^{40} \mathrm{Ar}+{ }^{45} \mathrm{Sc}[16]$ at $150 \mathrm{~A} \mathrm{GeV} / c$. Results for the correlator $\Delta F_{2}(M)$ are presented in Fig.2. In the case of $\mathrm{Be}+\mathrm{Be}$ system, Fig.2(d), $\Delta F_{2}(M)$ values fluctuate around zero, and no intermittency effect is observed. It should be noted that the average proton multiplicity for $\mathrm{Be}+\mathrm{Be}$ events in the mid rapidity range was $1.48 \pm 0.74$ [16], i.e. less than 2 protons on average per event; given such low multiplicity, intermittency analysis must be considered inconclusive as to the presence of a critical component.

In the case of the $\mathrm{Ar}+\mathrm{Sc}$ system, the available data were partitioned into three subsets corresponding to $0-5 \%, 5-10 \%$ and $10-15 \%$ most central collisions, determined by projectile spectator energy. Protons were selected with a minimum purity of $90 \%$, where purity quantifies the probability that the candidate particle is a proton. As can be seen in Fig.2(e-g), central (0-5\%) Ar+Sc data show no intermittency, whereas in mid-central (5-10\%, 10-15\%) Ar+Sc we see a tendency for increased $\Delta F_{2}(M)$ scaling with increased peripherality. It must be noted, however, that a power-law fit for $\phi_{2}$ cannot be performed on these data without properly taking into account bin correlations of $M$ values, and so a confidence interval for $\phi_{2}$ is presently unavailable. The solid red lines in Fig.2(e-g) are simply power-law scaling functions to guide the eye.
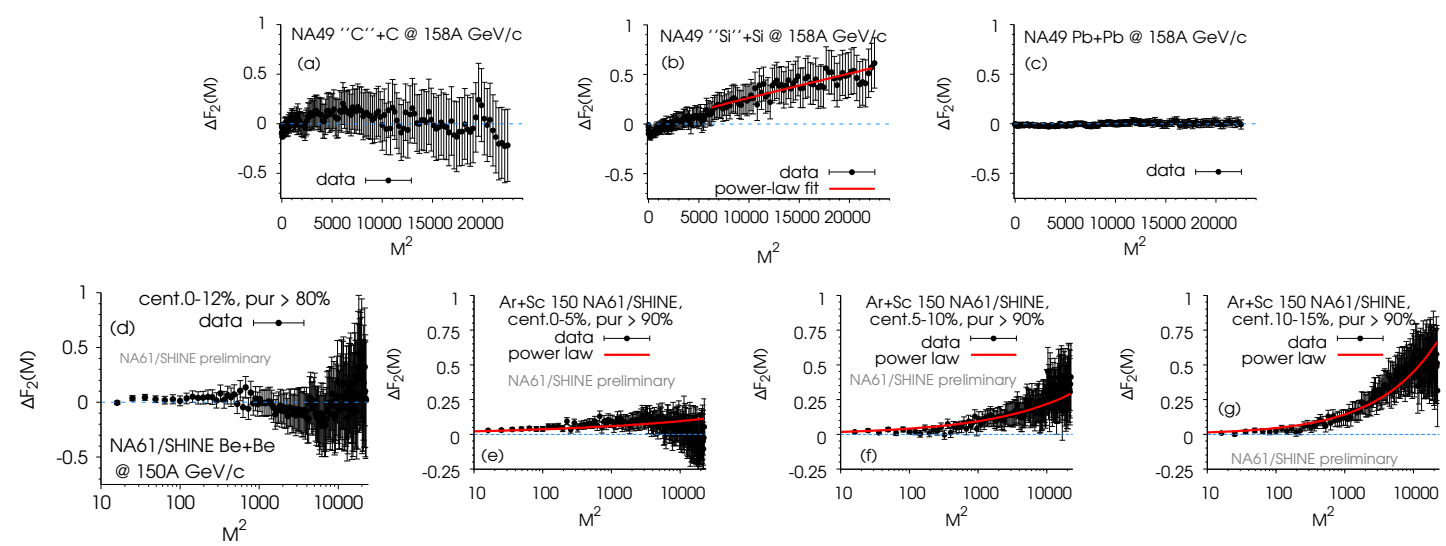

Figure 2: $\Delta F_{2}(M)$ for various experimental collisions at top SPS beam momentum. Top row: NA49 (a) "C" $+\mathrm{C}$ and $(b)$ "Si" $+\mathrm{Si}(0-12 \%$ most central), and (c) $\mathrm{Pb}+\mathrm{Pb}$ collisions ( $0-10 \%$ most central) at $158 \mathrm{~A} \mathrm{GeV/c}$ (Ref.[1]); Bottom row: NA61/SHINE (d) Be+Be (0-12\% most central) collisions [15], as well as (e) Ar+Sc collisions at $0-5 \%,(f) 5-10 \%$, and $(g) 10-15 \%$ centrality at $150 \mathrm{~A} \mathrm{GeV/c}$ [16]. 


\subsection{Statistical significance of NA61/SHINE Ar+Sc $\Delta F_{2}(M)$ effect}
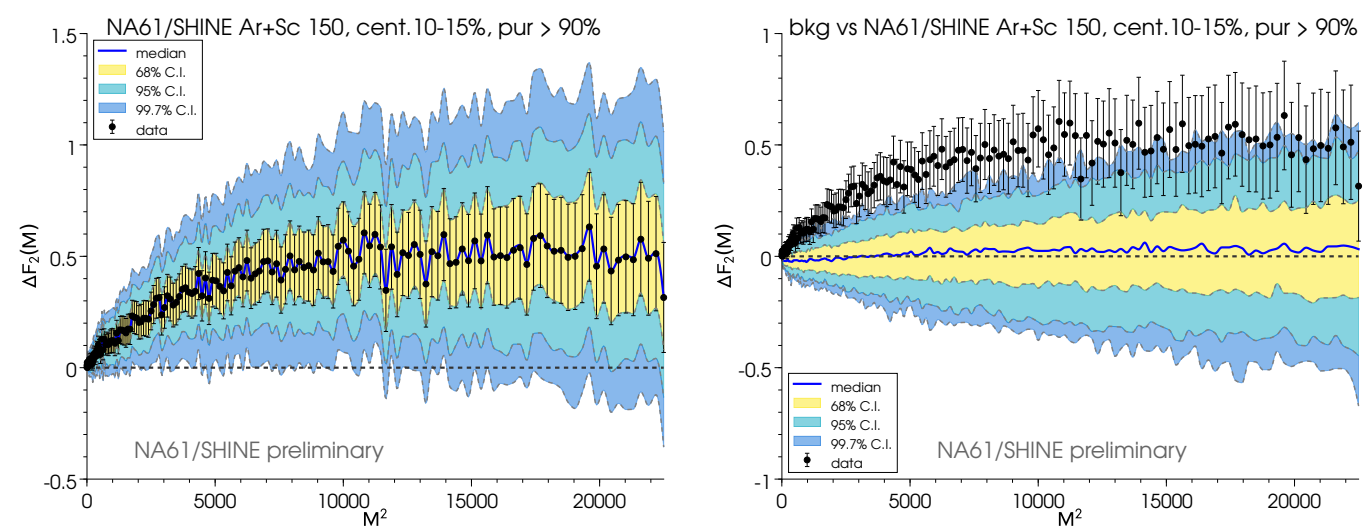

Figure 3: Left: $\Delta F_{2}(M)$ original sample values for $10-15 \%$ central $\mathrm{Ar}+\mathrm{Sc}$ collisions at $150 \mathrm{~A} \mathrm{GeV/c}$ (black points); error bars correspond to bootstrap standard error; colored bands indicate bootstrap confidence intervals; solid blue line gives the median value of bootstrap samples. Right: The same experimental $\Delta F_{2}(M)$ values (black points) compared to the $\Delta F_{2}(M)$ results for simulated random background protons.

As mentioned, the uncertainties involved in the $\Delta F_{2}(M)$ calculation are large; we therefore attempt to quantify the statistical significance of the non-zero effect we see in NA61/SHINE $\mathrm{Ar}+\mathrm{Sc} @ 150 \mathrm{~A} \mathrm{GeV} / c$ data by looking at the bootstrap distributions of $\Delta F_{2}(M)$ values.

Figure 3 (left) shows the values of $\Delta F_{2}(M)$ for $10-15 \%$ central $\mathrm{Ar}+\mathrm{Sc}$ collisions at $150 \mathrm{~A} \mathrm{GeV} / c$; original sample data values and their bootstrap standard errors are plotted against confidence intervals $(68-95-99.7 \%)$ of the $\Delta F_{2}(M)$ distributions obtained from 1000 bootstrap samples. Figure 3 (right) compares the experimental $\Delta F_{2}(M)$ values against the $\Delta F_{2}(M)$ values obtained from an uncorrelated proton background with the same inclusive characteristics as the original $\mathrm{Ar}+\mathrm{Sc}$ events. Fig. 3 indicates that random background can imitate an effect as large as seen in $\mathrm{Ar}+\mathrm{Sc}$ in about $\sim 5 \%$ of all cases, and the $\mathrm{Ar}+\mathrm{Sc}$ effect is above zero in $\sim 95 \%$ of bootstrap samples. Based on these findings, we tentatively assign a $95 \%$ statistical significance to the observed experimental result being not a purely random fluctuation.

\section{Summary and conclusions}

Intermittency analysis of proton density fluctuations in transverse momentum space provides us with a promising set of observables for the detection of the critical point of strongly interacting matter. The intermittency analysis performed on NA49 "Si"+Si data at the maximum SPS energy already suggests the presence of a critical proton component with an estimated intermittency index value of $\phi_{2, B}=0.96_{-0.25}^{+0.38}$, overlapping with the critical QCD prediction, whereas no intermittency is observed in either the smaller " $\mathrm{C}$ " $+\mathrm{C}$ or the larger $\mathrm{Pb}+\mathrm{Pb}$ system at the same collision energy. The preliminary analysis of the NA61/SHINE central Be+Be system at $150 \mathrm{~A} \mathrm{GeV/c}$ consistently show no positive result.

We see the first indication of a non-trivial intermittency effect in NA61/SHINE, in our preliminary analysis of the SSFMs $\Delta F_{2}(M)$ of $\mathrm{Ar}+\mathrm{Sc}$ collisions at $150 \mathrm{~A} \mathrm{GeV} / c$. The significance of the 
effect seems to increase for less central collisions in the case of proton purity thresholds of $90 \%$ and above. However, due to the magnitude of SSFMs uncertainties, and the fact that $F_{2}(M)$ values for distinct $M$ are correlated, the quality of $\Delta F_{2}(M)$ power-law scaling remains still to be established, and an estimation of $\phi_{2}$ confidence intervals is still pending.

A continued, final analysis of the total available statistics of NA61/SHINE Ar+Sc data, and its extension to other system sizes (Xe+La) and energies of the NA61/SHINE program will hopefully lead to an accurate determination of the critical point location.

Acknowledgments: This work was supported by the National Science Centre, Poland (grant no. 2014/14/E/ST2/00018).

\section{References}

[1] T. Anticic et al, Eur. Phys. J. C 75: 587 (2015).

[2] A. Aduszkiewicz et al. [NA49-future Collab.], CERN-SPSC-2006-034.

[3] M. Gazdzicki, P. Seyboth, Acta Phys. Pol. B 47, 1201 (2016) and references therein.

[4] N. G. Antoniou, Y. F. Contoyiannis, F. K. Diakonos, A. I. Karanikas and C. N. Ktorides, Nucl. Phys. A 693, 799 (2001).

[5] Y. Hatta and M. A. Stephanov, Phys. Rev. Lett. 91, 102003 (2003).

[6] N. G. Antoniou, Y. F. Contoyiannis, F. K. Diakonos and G. Mavromanolakis, Nucl. Phys. A 761, 149 (2005).

[7] N. G. Antoniou, F. K. Diakonos, A. S. Kapoyannis and K. S. Kousouris, Phys. Rev. Lett. 97, 032002 (2006).

[8] T. Vicsek, Fractal Growth Phenomena (World Scientific, Singapore, 1989). ISBN 9971-50-830-3.

[9] J. Wosiek, Acta Phys. Polon. B 19, 863 (1988).

[10] A. Białas and R. C. Hwa, Phys. Lett. B 253, 436 (1991).

[11] W. J. Metzger, "Estimating the Uncertainties of Factorial Moments", HEN-455 (2004).

[12] B. Efron, Ann. Stat. 7, 1 (1979).

[13] T. Hesterberg et al., Bootstrap Method and Permutation Tests (W. H. Freeman \& Co., USA, 2003), ISBN-10:0716757265.

[14] C. Michael, Phys. Rev. D 49, 2616 (1994).

[15] N. Davis et al., for the NA61/SHINE Collaboration, PoS (CPOD2017) 054 (2018).

[16] N. Davis et al., for the NA61/SHINE Collaboration, Acta Phys. Polon. B 50, 1029 (2019). 